\title{
Boston Bowel Preparation Segment Score
} 3

National Cancer Institute

\section{Source}

National Cancer Institute. Boston Bowel Preparation Segment Score 3. NCI Thesaurus.

Code C138210.

Entire mucosa of colon segment seen well with no residual staining, small fragments of stool or opaque liquid. 